\title{
COMPUTATIONAL FLUID-STRUCTURE INTERACTION FRAMEWORK FOR SIMULATING CHARACTERISTIC DEFORMATIONS IN INSECT FLAPPING WINGS
}

\author{
MINATO ONISHI ${ }^{*}$ AND DAISUKE ISHIHARA ${ }^{\dagger}$ \\ * Department of Computer Science and System Engineering \\ Kyushu Institute of Technology \\ 680-4 Kawazu, Iizuka, Fukuoka, 820-8502, Japan \\ e-mail: oonishi-m@solid.mse.kyutech.ac.jp,www-solid.mse.kyutech.ac.jp \\ ${ }^{\dagger}$ Department of Intelligent and Control Systems, Kyushu Institute of Technology \\ Kyushu Institute of Technology \\ 680-4 Kawazu, Iizuka, Fukuoka, 820-8502, Japan \\ e-mail: ishihara@mse.kyutech.ac.jp,www-solid.mse.kyutech.ac.jp
}

Key words: Fluid-Structure Interaction, Insect Flapping Wing, Cambering, Feathering, Pixel Wing Model, Projection Method, Dynamic Similarity Law.

\begin{abstract}
In this study, a computational fluid-structure interaction (FSI) framework for characteristic deformations in insect's wings is proposed. The proposed framework consists of a pixel wing model using a structured shell finite element mesh, a projection method for the monolithic FSI monolithic equations using an algebraic splitting, and the FSI dynamic similarity law to measure dynamic similarity between model's and actual insect's flights. It is shown that the proposed framework can directly simulate passive feathering and cambering in insect's wings caused by the FSI, whose magnitudes are very close to those of actual insects.
\end{abstract}

\section{INTRODUCTION}

In insect's wings, wing membranes are supported by a complicated network of veins. Characteristic wing shape changes such as feathering and cambering are observed in many species of insects. These changes will be the elastic deformation caused by the inertial and aerodynamic forces because of no interior muscle in insects' wings [1]. Many literatures have reported their essential importance for creating insect's flight abilities [2-5]. However, the detailed mechanism of these characteristic deformations is still unclear because of the complicated wing's structure strongly coupled with the surrounding air flow.

In this study, a computational FSI framework for simulating characteristic deformations in insect flapping wings is proposed. The proposed framework consists of a pixel wing model using a structured shell finite element mesh [6], a projection method for the FSI monolithic equation system using an algebraic splitting [7], and the FSI dynamic similarity law to measure dynamic similarity between model's and actual insect's flights [8]. It is shown that the proposed framework can simulate the passive feathering and cambering caused by the FSI directly, whose magnitudes are very close to those of actual insects. 


\section{PIXEL MODELING OF INSECT FLAPPING WING}

\subsection{Pixel wing model}

The proposed wing model consists of a structured mesh using shell finite elements as shown in Fig. 1. Each element represents a pixel. The complicated network of veins is simplified by the leading edge, the center vein, and the root vein pixels based on Ref. [9] that represent the functionally distinct domains as the supporting structures. The rest of the pixels represents the wing membrane. The different set of the pseudo-elastic material properties is given to the pixels in each domain to represent the different mechanical behavior of the leading edge, the center vein, the root vein, and the wing membrane. The leading edge governs the flexural rigidity of the wing's span-wise direction $G_{\mathrm{s}}$. Hence, the Young's modulus of the leading edge is determined such that the flexural rigidity is equal to $G_{\mathrm{s}}$ of an actual insect's wing [10]. The root vein governs the flexural rigidity of the wing's chord-wise direction $G_{\mathrm{c}}$. Hence, the Young's modulus of the root vein is determined such that the flexural rigidity is equal to $G_{\mathrm{c}}$ of an actual insect's wing [10]. The Young's modulus of the center vein is determined such that the flexural rigidity is equal to that of a most significant vein in the center domain of an actual insect's wing $[11,12]$. The Young's modulus of the wing membrane $E_{\mathrm{m}}$ is determined from an actual insect's cuticle [13].

\subsection{Modeling of the insect flapping motion}



\section{Fig. 2 shows the schematic} plane with the stroke an displacement $\varphi$, which The trapezoidal functio actual insects [14] as shown


and deceleration time $t_{\varphi}$ is set to $T_{\varphi} / 8$, which is a typical value. This time history is applied to

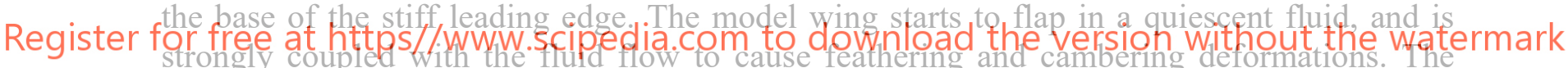

feathering motion can be described using the feathering angular displacement $\theta$, which is positive in the counter-clockwise direction about the torsional axis. The magnitude of the camber is defined as the ratio of the wing's height $C_{\mathrm{h}}$ to the wing's chord length $C_{1}$ as shown in Fig. 4, and the sign of the camber is positive if the shape of the camber is concave along the direction of the flapping translation [15].

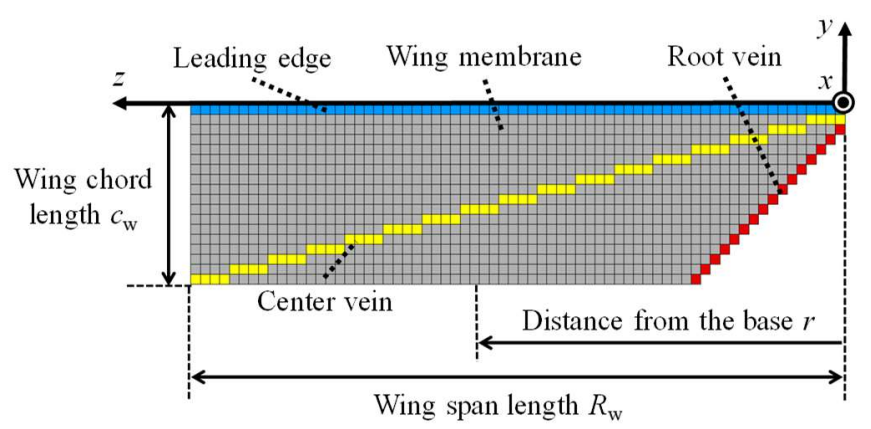

Figure 1: Pixel wing model

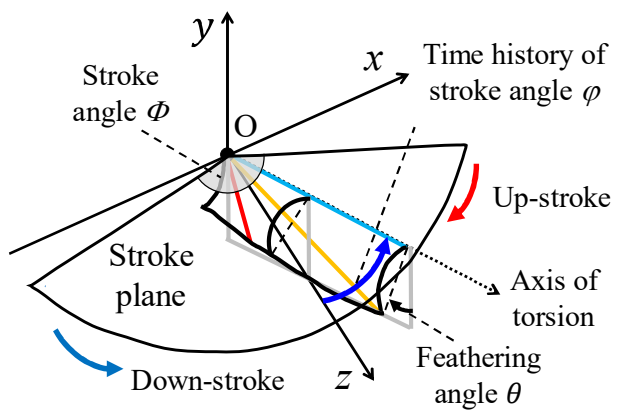

Figure 2: Schematic of the flapping motion 


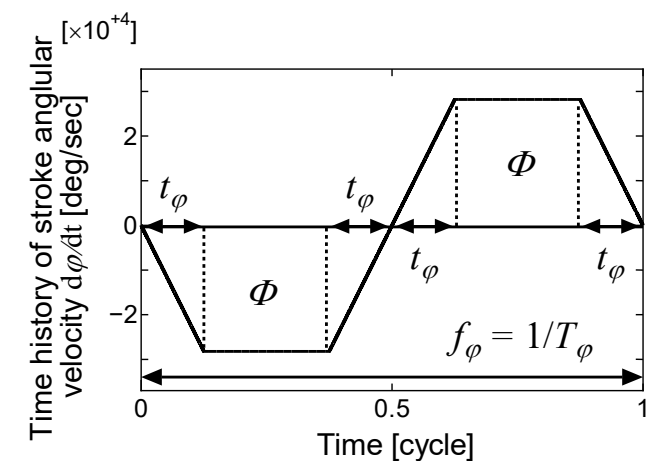

Figure 3: Time history of the stroke angular velocity

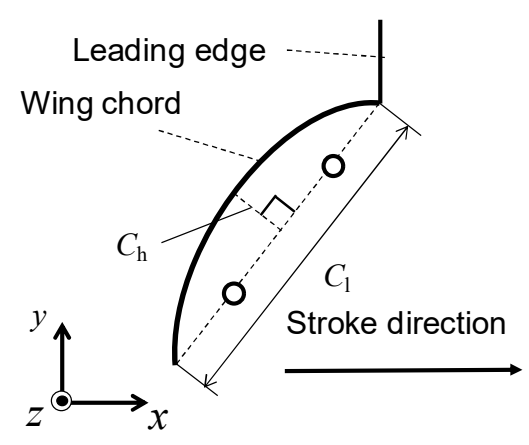

Figure 4: Definition of the camber

\section{FLUID-STRUCTURE INTERACTION ANALYSIS OF THE WING MODEL}

\subsection{Governing equations}

The equilibrium equation for the elastic body can describe the deformable wing as follows:

$$
\rho^{\mathrm{s}} \frac{d^{2} u_{i}^{\mathrm{s}}}{d t^{2}}=\frac{\partial \sigma_{i j}^{\mathrm{s}}}{\partial x_{j}}
$$

where $\partial / \partial t$ is the ALE time derivative, the superscript $\mathrm{f}$ stands for a fluidic quantity, and $v^{\mathrm{m}}{ }_{i}$ is the $i$ th component of the velocity vector of the ALE coordinate.

The following conditions are satisfied on the interface between the wing and the fluid:

$$
v_{i}^{\mathrm{f}}=v_{i}^{\mathrm{s}}, \sigma_{i j}^{\mathrm{f}} n_{j}^{\mathrm{f}}+\sigma_{i j}^{\mathrm{s}} n_{j}^{\mathrm{s}}=0,
$$

where $n_{i}^{\mathrm{f}}$ and $n^{\mathrm{s}}{ }_{i}$ are the $i$ th components of the outward unit normal vectors on the fluidstructure interface corresponding to the fluid and the structure, respectively.

\subsection{Projection method for the monolithic fluid-structure interaction system}

Eqs. (1) and (2) were discretized using the finite element method, and combined using the interface conditions (3) to obtain a monolithic equation system for the FSI as follows:

$$
{ }_{\mathrm{L}} \mathbf{M a}+\mathbf{C v}+\mathbf{N}+\mathbf{q}(\mathbf{u})-\mathbf{G p}=\mathbf{g},{ }_{\tau} \mathbf{G v}=\mathbf{0},
$$


where $\mathbf{M}, \mathbf{C}$, and $\mathbf{G}$ are the mass, diffusive, and divergence operator matrices, respectively, and $\mathbf{N}, \mathbf{q}, \mathbf{g}, \mathbf{a}, \mathbf{v}, \mathbf{u}$, and $\mathbf{p}$ are the convective term, elastic internal force, external force, acceleration, velocity, displacement, and pressure vectors, respectively. The subscript L represents the lumping, and the superscript $\tau$ indicates the transpose.

The monolithic method solves Eqs. (4a) and (4b) simultaneously, and it can satisfy the interface conditions (3) implicitly. Hence, it can avoid spurious numerical power on the interface, which yields numerical instability. However, this formulation leads to an illconditioned system of equations. Therefore, a projection method using algebraic splitting was used to avoid this difficulty [7]. The monolithic equations system (4) was linearized and split into equilibrium equations and a pressure Poisson equation as follows:

$$
\begin{aligned}
& \mathbf{M}^{*} \Delta \hat{\mathbf{a}}=\Delta \mathbf{g}, \\
& \gamma \Delta t_{\tau} \mathbf{G}_{\mathrm{L}} \mathbf{M}^{-1} \mathbf{G} \Delta \mathbf{p}=-{ }_{\tau} \mathbf{G} \hat{\mathbf{v}}, \\
& \mathbf{M}^{*} \Delta \mathrm{a}-\mathrm{G} \Delta \mathrm{p}=\Delta \mathrm{g},
\end{aligned}
$$

where $\hat{\mathbf{a}}$ is the intermediate acceleration, $\hat{\mathbf{v}}$ is the intermediate velocity, $\mathbf{M}^{*}$ is the generalized mass matrix, which consists of the lumped mass and tangential stiffness matrices, $\Delta$ and $t$ are the variable increment and time, respectively, and $\Delta \mathbf{g}$ is the residual vector of (4a). Note that the relationships among the state variables based on the Newmark's method are used in these equations. In the nonlinear iteration of each time step, first, the equilibrium equation (5) is


solved to derive the intermediate velocity fie
solved to derive the current pressure field p
incompressibility constraint (4b), and, findly,
the current velocity field v.


4 NUERICAL EXAMIPLE

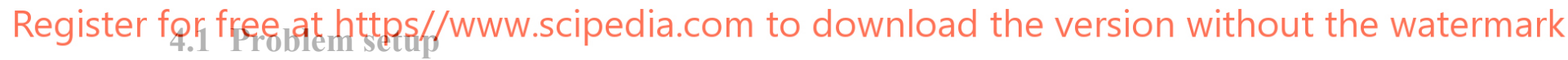

Fig. 5 shows the finite element meshes of the surrounding fluid domain. The wing is modeled using mixed interpolation of tensorial components shell elements [16] (number of nodes: 1,191; number of elements: 1,104$)$, while the fluid domain is modeled using stabilized linear equal-order-interpolation velocity-pressure elements (number of nodes: 276,138; number of elements: $1,578,960$ ). The wing's geometric parameters, the material properties of the wing's membrane, and the kinematic parameters of the flapping motion are given based on the data obtained from the actual insects $[11,12,14,17,18]$. The parameters used in this study are summarized as follows: The wing span length $R_{\mathrm{w}}=0.0113 \mathrm{~m}$, the wing chord length $c_{\mathrm{w}}=0.00311 \mathrm{~m}$, the wing's thickness $t=2.0 \mu \mathrm{m}$ (constant throughout the entire wing), the Young's modulus of wing membrane $=7.0 \mathrm{GPa}$, the flexural rigidity of along the wing's span-wise direction $G_{\mathrm{s}}=4.0 \mu \mathrm{Nm}^{2}$, the flexural rigidity of along the wing's chord-wise direction $G_{\mathrm{c}}=0.35 \mu \mathrm{Nm}^{2}$, the flexural rigidity of the most significant center vein $=0.26$ $\mathrm{nNm}^{2}, E_{\mathrm{m}}=7.0 \mathrm{GPa}$, Poisson's ratio $v=0.49, \Phi=71^{\circ}$, the flapping frequency $f_{\varphi}\left(=1 / T_{\varphi}\right)=$ $148 \mathrm{~Hz}$, the fluid mass density $\rho^{\mathrm{f}}=1.205 \mathrm{~kg} / \mathrm{m}^{3}$, the fluid viscosity $\mu^{\mathrm{f}}=1.822 \times 10^{-5} \mathrm{~Pa} \mathrm{~s}$, the time increment $\Delta t=T_{\varphi} / 150000$. $\Phi$ is corrected to satisfy the dynamic similarity law for fluidstructure interaction [8]. 
(a)



(b)

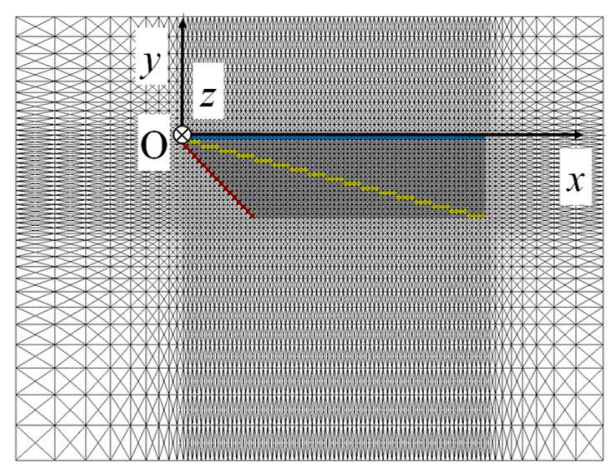

Figure 5: Finite element mesh of the surrounding fluid. (a) the bird eye view, and (b) the $y z$ plane view.

\subsection{Results and discussion}

Fig. 6 shows the time history of the feathering angle $\theta$. The normalized position is defined as $r / R_{\mathrm{w}}$, where $r$ is the distance from the wing base. As shown in this figure, the feathering motion is adequately caused by the FSI. The magnitude of $\theta$ in the case of $r / R_{\mathrm{W}}=1.0$ is larger than that in the case of $r / R_{\mathrm{W}}=0.5$. This is because the wing's flapping speed at $r / R_{\mathrm{W}}=1.0$ larger than that at $r / R_{\mathrm{W}}=0.5$ leads to the dynamic pressure acting on the wing at $r / R_{\mathrm{W}}=1.0$ larger than that at $r / R_{\mathrm{W}}=0.5$. The maximum $\theta$ in the case of $r / R_{\mathrm{W}}=1.0$ is $36^{\circ}$, which is close

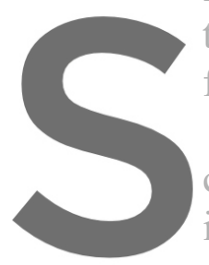
to the actual observations in insects [14]. It follows from these results that the framework can simulate the passive feathering motion directly

Fig. 7 shows the deformation of the wing mod cambering motion is adequately caused by the is close to the actual observation in insects proposed framework can simulate the passive cambering motion directly.

Register for free at https//www.scipedia.com to download the version without the watermark



Figure 6: Time history of the feathering angle.

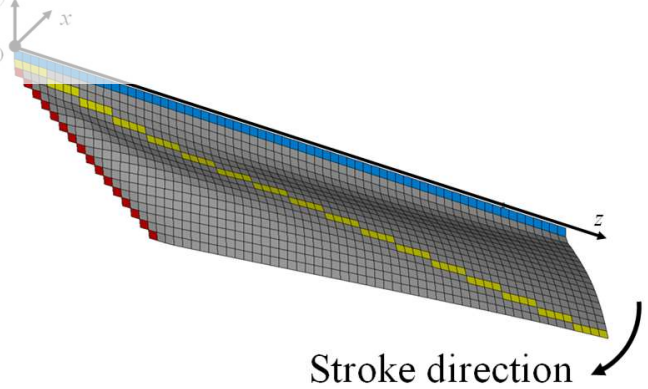

Figure 7: Deformation of the wing model at 0.25 cycle.

\section{CONCLUDING REMARKS}

In this study, a computational FSI framework for the characteristic deformations in insect flapping wings was proposed. The passive feathering and cambering simulated by the proposed framework were close to those observed in actual insects. Therefore, the proposed framework can simulate the characteristic deformations of insect's flapping wings directly. As long as we know, this is the first study that directly simulates both the feathering and 
cambering of the flapping wing caused by the FSI, which are close to the actual observations in insects. Therefore, the proposed framework will contribute to revealing passive mechanisms in insect's flapping wings.

\section{REFERENCES}

[1] R. J. Wooton, R. C. Herbert, P. G. Young and K. E. Evans: Approaches to the structural modeling of insect wings. Philosophical Transactions of the Royal Society of London B, 358, pp. 15771587 (2003).

[2] J. Young, S. M. Walker, R. J. Bomphrey, G. K. Taylor and A. L. R. Thomas: Details of insect wing design and deformation enhance aerodynamic function and flight efficiency, Science, 325, pp.1549-1552 (2009).

[3] G. Du and M. Sun, "Effects of the wing deformation on the aerodynamic forces in hovering hoverflies", The Journal of Experimental Biology, 213, pp. 2273-2283 (2010).

[4] T. Nakata and H. Liu: "A fluid-structure interaction model of insect flight with flexible wings", Journal of Computational Physics, 231, pp. 1822-1847 (2012).

[5] T. Nakata, R. Noda and H. Liu: "Effect of twist, camber and spanwise bending on the aerodynamic performance of flapping wings", Journal of Biomechanical Science and Engineering, 13, 2 (2018).

[6] M. Onishi and D. Ishihara: "Modeling the cambering of the flapping wings of an insect using rectangular shell finite elements", Journal of Advance Simulation in Science and Engineering, 7, 1, 181-188 (2020)

[7] D. Ishihara and T incompressible fluid Engineering and Scien

\section{Ishihara, T. Horie \\ structure interaction}

Experimental Biolog
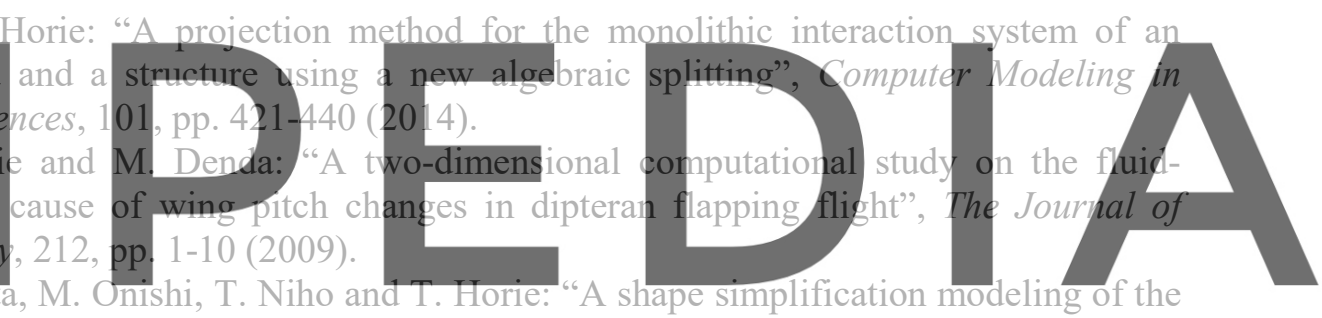
cambering in insect's flapping wings using beam and shell", Transactions of the Japan Society



[10] S. A. Combes and T. L. Daniel: "Flexural stiffness in insect wings I. Scaling and the influence of the wing venation", The Journal of Experimental Biology, 206, pp. 2979-2987, 2003.

[11] H. Tanaka, J. P. Whitney and R. J. Wood: "Effect of flexural and torsion wing flexibility on lift generation in hoverfly flight", Integrative and Comparative Biology, 51, pp. 142-150 (2011).

[12] S. A. Wainwright, W. D. Biggs, J. D. Currey, J. M. Gosline: Mechanical Design in Organism, Princeton University Press (1982).

[13] J. F. V. Vincent and U. G. K. Wegst: "Design and mechanical properties of insect cuticle", Arthropod Structure \& Development, 33, pp. 187-199, 2004.

[14] C. P. Ellington: "The aerodynamics of hovering insect flight III. Kinematics", Philosophical Transactions of the Royal Society of London B, 305, 1122, pp. 41-78 (1984).

[15] S. M. Walker, A. L. R. Thomas and G. K. Taylor: "Deformable wing kinematics in free-flying hoverfly", Journal of the Royal Society Interface, 7, pp. 131-142 (2010).

[16] H. Noguchi and T. Hisada: "Sensitivity analysis in post-buckling problems of shell structures", Computers and Structures, 47, pp. 699-710 (1993).

[17] H. Tanaka and R. J. Wood: "Fabrication of corrugated artificial insect wings using laser micromachined molds", Journal of Micromechanics and Microengineering, 20, 075008 (2010).

[18] A. R. Ennos: "The kinematics and aerodynamics of the free flight of some Diptera", The Journal of Experimental Biology, 142, pp. 49-85 (1989). 